\title{
1-S01-4 Symposium
}

\section{Central regulation mechanisms for stress-induced frequent urination}

\author{
Takahiro Shimizu $^{1}$, Shogo Shimizu ${ }^{1}$, Youichirou Higashi ${ }^{1}$, Naoki Yoshimura $^{2}$, Motoaki Saito $^{1}$
}

${ }^{I}$ Dept. of Pharmacol., Kochi Med. Sch., Kochi Univ., ${ }^{2}$ Dept. of Urol., Univ. Pittsburgh Sch. Med.

Psychological stress exacerbates symptoms of bladder dysfunction including overactive bladder and bladder pain syndrome/interstitial cystitis not only in rodent models but also in human patients. Bombesin (BB)-related peptides and $\mathrm{BB}$ receptors in the brain have been implicated in the mediation/integration of stress responses. We have found that brain $\mathrm{BB}$ induces frequent urination through the BB receptors, serotoninergic nervous system and corticotropinreleasing factor $(\mathrm{CRF})$ receptors. Interestingly, the $\mathrm{BB}$-induced response is independent of the BB-induced activation of the sympatho-adrenomedullary outflow, one of the components of the primary systems for maintaining or reinstating homeostasis during stress exposure. These findings indicate that brain BB, 5-HT and CRF receptors could be new therapeutic targets for bladder dysfunction exacerbated by stress exposure. There are several concepts regarding central regulation mechanisms for the bladder function in the normal and pathological conditions. However, the exact brain pathophysiological mechanisms underlying stress-induced effects on the bladder are largely unknown. Therefore, our findings could pioneer a novel neuropharmacological field, central "Neuro-UroPharmacology". 